\title{
In Silico Investigation of the Thyroid Hormone Activity of Hydroxylated Polybrominated Diphenyl Ethers
}

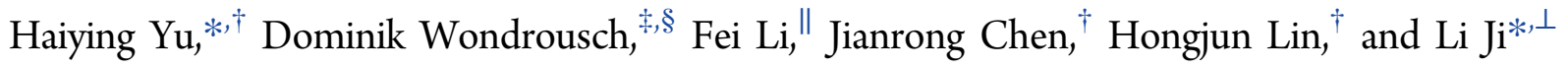 \\ ${ }^{\dagger}$ College of Geography and Environmental Sciences, Zhejiang Normal University, Yingbin Avenue 688, 321004 Jinhua, P.R. China \\ ${ }^{\ddagger}$ UFZ Department of Ecological Chemistry, Helmholtz Centre for Environmental Research, Permoserstrasse 15, 04318 Leipzig, \\ Germany \\ ${ }^{\S}$ Institution for Organic Chemistry, Technical University Bergakademie Freiberg, Leipzig Strasse 29, 09596 Freiberg, Germany \\ "Yantai Institute of Coastal Zone Research, Chinese Academy of Science, Chunhui Road 17, 264003 Yantai, P.R. China \\ ${ }^{\perp}$ College of Environmental and Resource, Zhejiang University, Yuhangtang Road 866, 310058 Hangzhou, P.R. China
}

Supporting Information

ABSTRACT: Polybrominated diphenyl ethers (PBDEs) have been shown to have a disruptive effect on the thyroid hormone system, and one possible mechanism is the direct binding of their hydroxylated metabolites (HO-PBDEs) to thyroid hormone receptors (TRs). However, the experimental data on the thyroid hormone activity of HO-PBDEs are limited, and the molecular interaction mechanism remains unclear, impeding the ecological risk assessment for these widespread contaminants. In the present research, a quantum chemical approach was developed to predict the thyroid hormone activity of HO-PBDEs using the electronic structure parameters of neutral molecules. The ab initio HF/6-31G** algorithm was employed to optimize the molecular geometry and to calculate local molecular parameters regarding effective energy and electron transfer amount. The mechanistic analysis shows that the ability of the hydroxyl oxygen and hydrogen atom to donate or accept additional electron charges is an important property

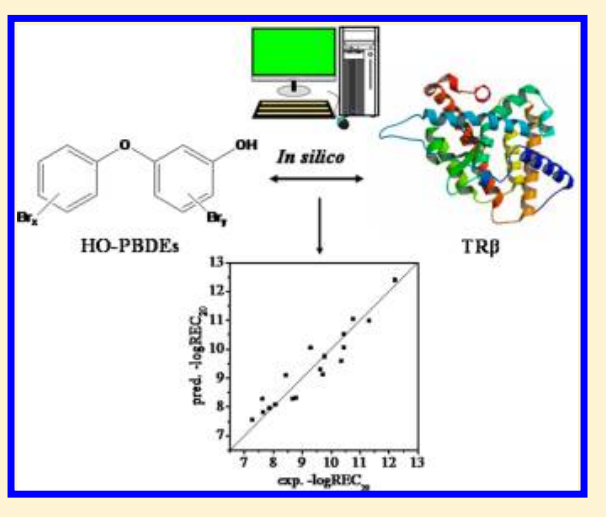
affecting the chemical activity of the thyroid hormone. The derived regression model was shown to have a good statistical performance and could be used to predict the thyroid hormone activity of other HO-PBDE congeners for which experimental measurements are not possible or are restricted. Therefore, the model has the potential to be a useful tool in the application of integrated testing strategies.

\section{INTRODUCTION}

Polybrominated diphenyl ethers (PBDEs) were originally designed as essentially inert flame retardants, and their entry into the environment has resulted in widespread contamination levels in abiotic and biotic categories. ${ }^{1-4}$ The endocrine disruption effect of PBDEs has arisen in the past decade and is currently of high concern. ${ }^{5,6}$ More recently, in vitro tests have revealed that the metabolic conversion of PBDEs to hydroxylated PBDEs (HO-PBDEs) appears to be a major cause for their potency as endocrine disruptors. ${ }^{7,8}$ HO-PBDEs have also been detected in samples of air, ${ }^{9}$ water, and precipitation, ${ }^{10}$ algae and mussels, ${ }^{11}$ fish, ${ }^{12,13}$ birds,${ }^{14}$ terrestrial animals, ${ }^{15}$ and in human blood ${ }^{16}$ and breast milk. ${ }^{17}$ These halogenated phenolic compounds could be produced from the parent compounds (PBDEs) through interactions with the hydroxyl group in the atmosphere ${ }^{18}$ and/or biotransformation catalyzed by the active center of the P450 enzyme in organisms. ${ }^{19}$ HO-PBDEs have received substantial attention because of their severe biological effects. ${ }^{20}$

Because of the structural resemblance to thyroid hormones (THs), HO-PBDEs could disturb the thyroid hormone homeostasis of experimental animals ${ }^{21-23}$ and humans. ${ }^{5,24}$ In vitro tests have indicated that one possible mechanism of HOPBDE interference with thyroid hormones is for the HOPBDEs to act as potent competitors for binding to transport proteins such as transthyretin (TTR $)^{25}$ and/or directly binding to thyroid hormone receptors (TRs), ${ }^{26}$ thereby displacing the natural ligand and changing the normal level of thyroid hormones in the bloodstream, consequently disturbing thyroid hormone homeostasis. Therefore, HO-PBDEs have attracted increasing attention. ${ }^{27}$

TRs are members of the nuclear receptor superfamily that functions in ligand-dependent transcription, and they play an important role in the regulation of metabolism and heart rate as well as in the development of organisms. ${ }^{28}$ TRs have two gene expressions, TR $\alpha$ and $\operatorname{TR} \beta$, and $\operatorname{TR} \beta$ mediates thyroid hormone effects on liver and other tissues. ${ }^{29}$ In a previous study, the hormone activities of 18 HO-PBDEs on human TR $\beta$ were measured, and hydrogen bonding and electrostatic interactions were revealed as critical molecular interactions that led to the thyroid hormone activities of HO-PBDEs. These

Received: March 25, 2015

Published: July 12, 2015 
Scheme 1. Molecular Structures of the Selected 18 HO-PBDEs

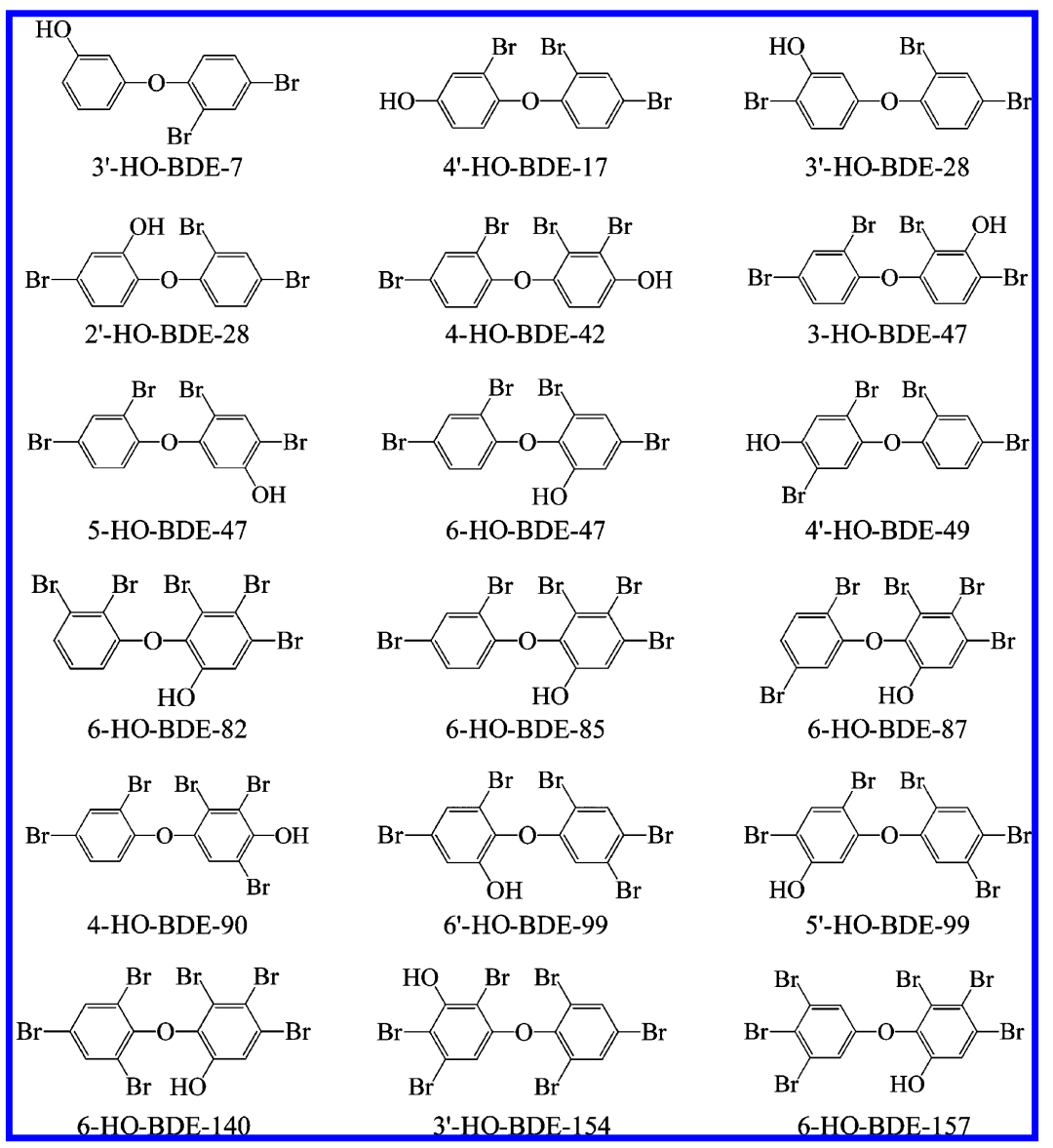

properties were revealed by employing molecular docking and by developing the quantitative structure-activity relationship (QSAR) model. ${ }^{20}$ Similar results were also obtained through the ligand- and receptor-based 3D-QSAR models. ${ }^{30}$ However, the model performance should be improved for predictive applications, and further analysis for the mechanism of activity is also required.

Quantum chemical descriptors are widely employed in QSAR models because they can express the electronic and geometric properties of molecules and their interactions that account for the proposed mechanism of action. ${ }^{31}$ In this research, a series of local molecular parameters calculated by ab initio methodology is employed to develop a new quantum chemical model to represent the interactions between $\operatorname{TR} \beta$ and HO-PBDEs. These descriptors were originally introduced by Klamt ${ }^{32,33}$ and were successfully applied to the estimation of the hydrogen bonding strength, ${ }^{34,35}$ a process observed between the hydroxyl groups of HO-PBDEs and the amino acid residues of $\operatorname{TR} \beta$, acting as a characteristic interaction for the thyroid hormone activity of HO-PBDE molecules. ${ }^{20,30}$ In addition, these parameters have also been shown capable of addressing the electrostatic interactions, ${ }^{34,35}$ another important molecular interaction that governs the activity of HO-PBDEs to $\operatorname{TR} \beta .^{20,30}$ Consequently, the local molecular descriptors are expected to be capable of quantifying the electronic interactions between HO-PBDEs and $\operatorname{TR} \beta$ and to explain the activity mechanism because of their explicit physicochemical meanings.

Site-specific reactivity extracts energy and electronic information that reflects the local characteristics of a given atomic site in the molecular environment. ${ }^{36-38}$ In this study, the site-specific reactivity is calculated at the hydroxyl group, which has been proven to be a critical site for binding of HOPBDEs with $\operatorname{TR} \beta$ to exert their disrupting activity. ${ }^{20,30}$ The statistical robustness and predictive ability of the derived model is tested by two validation strategies, and outliers are diagnosed by the Williams plot.

\section{MATERIALS AND METHODS}

Data Set and Chemical Structure. The experimental activity of the thyroid hormone in terms of $-\log \mathrm{REC}_{20}$ values (negative logarithm of the relative effective concentration (REC) inducing $20 \%$ of the maximum effect) was obtained from the literature. ${ }^{20}$ Eighteen data points of $-\log \mathrm{REC}_{20}$ values were measured by a recombinant two-hybrid yeast assay at $30^{\circ} \mathrm{C}$. The molecular structures of all HOPBDEs are shown in Scheme 1, and the $-\log \mathrm{REC}_{20}$ values are listed in Table 1.

Local Molecular Descriptors. The energy-weighted donor energy $E E_{\text {occ }}\left(E_{\text {ref }}, r\right)$ quantifies the electron donating ability of a molecule at atomic site $r$ and can be taken as a generalized site-specific highest occupied molecular orbital (HOMO) energy, with the following formula:

$$
\begin{aligned}
& E E_{\text {occ }}\left(E_{\text {ref }}, r\right)=\frac{\sum_{i=1}^{\mathrm{HOMO}} E_{i} \cdot w_{i}\left(E_{\text {ref }}, r\right)}{\sum_{i=1}^{\mathrm{HOMO}} w_{i}\left(E_{\text {ref }}, r\right)} \\
& \text { with } \\
& w_{i}\left(E_{\text {ref }}, r\right)=p_{i}(r) \exp \left(-\frac{E_{i}}{E_{\text {ref }}}\right) \quad p_{i}(r)=2 \sum_{\mu(r)} c_{\mu i}^{2}
\end{aligned}
$$

where the linear combination of atomic orbital-molecular orbital (LCAO-MO) coefficient $c_{\mu i}$ represents the contribution of the $\mu^{\text {th }} \mathrm{AO}$ 
Table 1. Experimental and Predicted - $\log \mathrm{REC}_{20}$ Values and the Calculated Descriptor Values Employed in Eq $6^{a}$

\begin{tabular}{|c|c|c|c|c|c|}
\hline \multirow[b]{2}{*}{ compds } & \multicolumn{2}{|c|}{$-\log \mathrm{REC}_{20}$} & \multirow[b]{2}{*}{$\begin{array}{c}Q E_{\text {occ }} \\
(-8.8 \mathrm{eV}, \mathrm{O})\end{array}$} & \multirow[b]{2}{*}{$\begin{array}{c}Q E_{\mathrm{vac}} \\
(3.8 \mathrm{eV}, \mathrm{H})\end{array}$} & \multirow[b]{2}{*}{$\begin{array}{l}\log \\
K_{\text {ow }}\end{array}$} \\
\hline & Exp. & Pred. & & & \\
\hline 3'-HO-BDE-7 & 7.64 & 7.82 & 0.429 & 3.767 & 4.51 \\
\hline $4^{\prime}-\mathrm{HO}-\mathrm{BDE}-17$ & 8.66 & 8.29 & 0.423 & 3.727 & 5.40 \\
\hline $2^{\prime}-\mathrm{HO}-\mathrm{BDE}-28$ & 8.07 & 8.09 & 0.425 & 3.633 & 5.40 \\
\hline $3^{\prime}-\mathrm{HO}-\mathrm{BDE}-28$ & 7.28 & 7.56 & 0.402 & 3.646 & 5.40 \\
\hline 4-HO-BDE-42 & 9.72 & 9.13 & 0.433 & 3.687 & 6.29 \\
\hline 3-HO-BDE-47 & 8.77 & 8.32 & 0.400 & 3.687 & 6.29 \\
\hline 5-HO-BDE-47 & 8.44 & 9.09 & 0.435 & 3.654 & 6.29 \\
\hline 6-HO-BDE-47 & 10.43 & 10.53 & 0.476 & 3.829 & 6.29 \\
\hline $4^{\prime}-\mathrm{HO}-\mathrm{BDE}-49$ & 7.87 & 7.96 & 0.391 & 3.633 & 6.29 \\
\hline 6-HO-BDE-82 & 10.44 & 10.05 & 0.507 & 3.086 & 7.18 \\
\hline 6-HO-BDE-85 & 9.77 & 9.75 & 0.502 & 3.016 & 7.18 \\
\hline 6-HO-BDE-87 & 9.29 & 10.05 & 0.506 & 3.091 & 7.18 \\
\hline 4-HO-BDE-90 & 7.63 & 8.28 & 0.374 & 3.639 & 7.18 \\
\hline $6^{\prime}-\mathrm{HO}-\mathrm{BDE}-99$ & 9.62 & 9.31 & 0.474 & 3.107 & 7.18 \\
\hline 5'-HO-BDE-99 & 10.34 & 9.59 & 0.427 & 3.652 & 7.18 \\
\hline 6-HO-BDE-140 & 11.31 & 10.98 & 0.447 & 3.732 & 8.07 \\
\hline $\begin{array}{l}3^{\prime} \text {-HO-BDE- } \\
154\end{array}$ & 10.76 & 11.06 & 0.455 & 3.681 & 8.07 \\
\hline 6-HO-BDE-157 & 12.2 & 12.40 & 0.471 & 4.047 & 8.07 \\
\hline
\end{tabular}

${ }^{a} Q E_{\text {occ }}(-8.8 \mathrm{eV}, \mathrm{O})$ is the energy-limited donor charge on the hydroxyl oxygen atom with energy penetration depth $\varepsilon=-8.8 \mathrm{eV}$ (eq $3)$, and $Q E_{\text {vac }}(3.8 \mathrm{eV}, \mathrm{H})$ is the energy-limited acceptor charge on the hydroxyl hydrogen atom with energy penetration depths $\varepsilon=3.8 \mathrm{ev}$ (eq 4). All $\log K_{\mathrm{ow}}$ values are calculated by EPI Suite, version $4.1 .^{40}$

at center $r$ to the $i$ th MO. Thus, the actual contribution of a given MO energy $E_{\mathrm{i}}$ to $E E_{\text {occ }}\left(E_{\text {ref }}, r\right)$ is evaluated by the extent of the local characteristics of the electron charge $\left(p_{i}\right)$ and the ratio of $E_{\mathrm{i}}$ to $E_{\mathrm{ref}}$. Correspondingly, the energy-weighted acceptor energy $E E_{\mathrm{vac}}\left(E_{\mathrm{ref}}, r\right)$ is defined by unoccupied $\mathrm{MO}$ energies and characterizes the capability of the molecule to accept additional electron charges at atomic site $r$.

Another local molecular parameter is the charge-limited donor energy $E Q_{o c c}(q, r)$, which is defined as follows:

$$
\begin{aligned}
& E Q_{\mathrm{occ}}(q, r)=\frac{1}{q} \sum_{i=1}^{\text {HOMO }} E_{i} w_{i}(q, r) \\
& \text { with }
\end{aligned}
$$

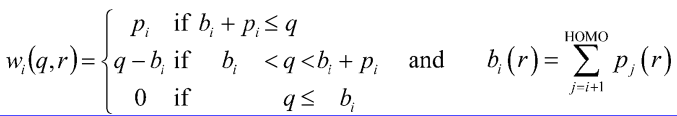

$E Q_{\text {occ }}(q, r)$ is calculated as the weighted mean of the occupied molecular orbital energies $E_{\mathrm{i}}$. The calculation begins from the HOMO and stops when the electron population $p_{i}$ at center $r$ reaches a predefined charge limit $q$. $E Q_{\mathrm{occ}}(q, r)$ approaches $E_{\mathrm{HOMO}}$ when $q$ is close to zero and decreases with an increasing amount of charge penetration depth $q$, which evokes an improvement of the localizability of $E Q_{o c c}$. Similarly, $E Q_{\text {vac }}(q, r)$ is defined by unoccupied MOs, characterizing the energy gain associated with accepting charge $q$ at atomic site $r$.

Complementary to these two descriptor pairs, energy-limited donor/acceptor charge $Q E_{\mathrm{occ}}(\varepsilon, r)$ and $Q E_{\mathrm{vac}}(\varepsilon, r)$ are designed to quantify the associated amount of charge released from or taken up at site $r$ with a given energy loss or gain. $Q E_{\text {occ }}(\varepsilon, r)$ is calculated by the following equation:

$$
Q E_{\mathrm{occ}}(\varepsilon, r)=\sum_{i=1}^{\text {HOMO }} p_{i}(r) w_{i}(\varepsilon)
$$

with

$$
w_{i}(\varepsilon)=\left\{\begin{array}{lll}
1 & \text { if } & \varepsilon \leq E_{i}-0.5 \\
E_{i}-\varepsilon+0.5 & \text { if } & E_{i}-0.5<\varepsilon<E_{i}+0.5 \\
0 & \text { if } \quad E_{i}+0.5 \leq \varepsilon
\end{array}\right.
$$

The parameter $\varepsilon$ is the energy penetration depth, which is used to limit the summation over the charge contribution $p_{i}(r)$ of the highest occupied MOs $i$ of AOs $\mu$ located at site $r$. Small $Q E_{\text {occ }}$ values indicate that the atomic sites have low electron donor ability. Correspondingly, the amount of accepted electron charge associated with the energy gain $\varepsilon$ is quantified by $Q E_{\text {vac }}(\varepsilon, r)$, which is represented as follows:

$$
\begin{aligned}
& Q E_{\mathrm{vac}}(\varepsilon, r)=\sum_{k=\mathrm{LUMO}}^{n} p_{k}(r) w_{k}(\varepsilon) \\
& \text { with } \\
& w_{k}(\varepsilon)=\left\{\begin{array}{rrl}
1 & \text { if } & E_{k}+0.5 \leq \varepsilon \\
E_{k}-\varepsilon+0.5 & \text { if } & E_{k}-0.5<\varepsilon<E_{k}+0.5 \\
0 & \text { if } \quad & \varepsilon \leq E_{k}-0.5
\end{array}\right.
\end{aligned}
$$

In addition, the $n$-octanol/water partition coefficient $\left(\log K_{\text {ow }}\right)$ is selected to characterize the partition of compounds between the biophase and the aqueous phase, according to the previous study. ${ }^{20}$

Quantum Chemical Calculations. The geometries of all molecules were optimized in their neutral electronic ground state in the gas phase employing the ab initio HF/6-31G** algorithm, which is implemented by the Gaussian 03 program package. ${ }^{39}$ Frequency analysis was performed on the same basis set to validate the energetic minima. Then, six local molecular descriptors $\left(E E_{\text {occ }}\left(E_{\text {ref }}, r\right), E E_{\text {vac }}\right.$ $\left(E_{\text {ref }}, r\right), E Q_{\text {occ }}(q, r), E Q_{\text {vac }}(q, r), Q E_{\text {occ }}(\varepsilon, r)$, and $\left.Q E_{\text {vac }}(\varepsilon, r)\right)$ were calculated at the hydroxyl group sites of HO-PBDEs according to eqs $1-4$. Reference energies $\left(E_{\mathrm{ref}}^{\mathrm{occ}}, E_{\mathrm{ref}}^{\mathrm{vac}}\right)$, charge penetration depths $\left(q_{\mathrm{oco}}\right.$ $\left.q_{\mathrm{vac}}\right)$, and energy penetration depths $\left(\varepsilon_{\mathrm{occ}}, \varepsilon_{\mathrm{vac}}\right)$ were optimized in a stepwise manner toward the $-\log \mathrm{REC}_{20}$ values. Furthermore, $\log K_{\mathrm{ow}}$ values were calculated using the EPI Suite, version $4.1 .^{40}$

Model Calibration. Multilinear regression (MLR) was performed to calibrate the prediction model employing SPSS 17.0. Squared correlation coefficient $r^{2}$ and predictive squared correlation coefficient $q^{2}$ were calculated to evaluate the calibration and predictive performance of the MLR model. ${ }^{41}$ Other statistical parameters, rootmean-square error $(\mathrm{rms})$, mean error $(\mathrm{me})$, systematic error (bias), maximum negative error (mne, largest underestimation), and maximum positive error (mpe, largest overestimation), were also calculated to further characterize the statistical performance.

Model Validation. The statistical robustness and predictive ability of the developed model were evaluated using simulated external validation and cross-validation. First, the entire data set was randomly divided into a temporary training set (70\% compounds) and a testing set ( $30 \%$ compounds), both covering the $-\log \mathrm{REC}_{20}$ value range and descriptor space. Then, a new regression model was recalibrated based on the $70 \%$ subset and was used to predict the $-\log \mathrm{REC}_{20}$ values of the $30 \%$ subset. The resulting $r^{2}$ and $q^{2}$ of the application revealed the prediction ability, and together with rms, provided further information about statistical robustness by comparing with $r^{2}$ and rms for recalibration (70\% subset).

The second validation strategy is the leave-10\%-out cross-validation. The entire data set was divided into 10 equiv subsets by stratified random selection. Then, nine subsets were used to develop a model that is employed to predict the one remaining subset; this process is repeated 10 times to ensure that every compound is evaluated. The resulting $q^{2}$ further represents the predictive ability and statistical robustness.

Influential Compounds and Outliers. Compounds with extreme descriptor values have a larger influence on the descriptor space and 
Table 2. Statistical Performance of Regression and Simulated External Validation for Predicting the $-\log$ REC ${ }_{20}$ of HO-PBDEs

\begin{tabular}{|c|c|c|c|c|c|c|c|c|}
\hline & $n$ & $r^{2}$ & $q^{2}$ & $\mathrm{rms}$ & bias & me & mpe & mne \\
\hline regression model & 18 & 0.902 & 0.902 & 0.427 & 0.001 & 0.358 & 0.760 & -0.750 \\
\hline training set & 13 & 0.888 & 0.888 & 0.462 & 0.001 & 0.388 & 0.670 & -0.840 \\
\hline test set & 5 & 0.936 & 0.921 & 0.362 & -0.122 & 0.350 & 0.360 & -0.450 \\
\hline
\end{tabular}

calibration of the regression coefficients than other compounds. Here, the leverage $h_{\mathrm{i}}$ of a given compound $i$ is taken as an indicator to identify high-influence compounds. The parameter $h_{\mathrm{i}}$ is the $i$ th diagonal element of the Hat matrix that relates the target values to their prediction counterparts, as follows: ${ }^{42}$

$$
h_{\mathrm{i}}=x_{\mathrm{i}}^{\mathrm{T}}\left(X^{\mathrm{T}} X\right)^{-1} x_{\mathrm{i}}
$$

where $X$ is a two-dimensional descriptor matrix comprising $n$ rows (compounds) and $p$ columns (descriptors), $X^{\mathrm{T}}$ is the transposition of $X,\left(X^{\mathrm{T}} X\right)^{-1}$ is the inverse of matrix $X^{\mathrm{T}} X$, and $x_{\mathrm{i}}$ is the descriptor rowvector of the query compound. An $h_{\mathrm{i}}$ larger than the warning leverage $h^{*}$ indicates that compound $i$ is distant from the descriptor space center and has a high influence on the model regression performance. ${ }^{42}$ Warning leverage $h^{*}$ equals $3 p / n$, where $p$ is the number of predictor variables, and $n$ is the number of observations.

A large gap exists between the calibrated values from regression models and the experimental values for the target property in the case of outliers; thus, a standardized residual greater than 3 or less than -3 is taken as an indicator for outliers. Influential compounds and outliers are visualized by a Williams plot of standardized prediction residuals versus leverage $h_{\mathrm{i}}$.

\section{RESULTS AND DISCUSSION}

Dataset Characteristics. As shown in Table 1 , the experimental - log $\mathrm{REC}_{20}$ values vary from 7.28 (3'-HO$\mathrm{BDE}-28)$ to 12.20 (6-HO-BDE-157), covering 5 orders of magnitude.

In this study, all compounds of interest are known to be monohydroxylated PBDEs with different numbers of bromine atoms. Among the 18 monohydroxylated PBDEs, one molecule is dibrominated $(6 \%)$, three are tribrominated $(17 \%)$, five are tetrabrominated $(28 \%)$, six are pentabrominated $(33 \%)$, and three are hexabrominated (17\%) HO-PBDEs (Scheme 1 and Table 1). The average $-\log \mathrm{REC}_{20}$ value for every group from di- to hexabrominated substitution is $7.64,8.00,9.05,9.52$, and 11.42 , indicating that the thyroid hormone activity increases with increasing number of bromine atoms in the HO-PBDE molecules.

Model Calibration. After the application of MLR analysis, the following regression model is obtained:

$$
\begin{aligned}
& -\log \mathrm{REC}_{20}=24.22 \times Q E_{\mathrm{occ}}(-8.8 \mathrm{eV}, \mathrm{O}) \\
& \quad+2.61 \times Q E_{\mathrm{vac}}(3.8 \mathrm{eV}, \mathrm{H})+0.79 \times \log K_{\mathrm{OW}}-15.96
\end{aligned}
$$

The developed model is a simple linear equation that contains two local descriptors located on the hydroxyl oxygen and hydroxyl hydrogen atoms, as well as $\log K_{\text {ow }}$, with good statistical performance reflected by $r^{2}=0.902$ and $\mathrm{rms}=0.427$ (Table 2). $r^{2}$ indicates that $90.2 \%$ of the $-\log \mathrm{REC}_{20}$ variance could be explained by the regression on two local reactivity parameters and $\log K_{\mathrm{ow}}$. The $-\log \mathrm{REC}_{20}$ values predicted by eq 6 and the descriptor values are listed in Table 1 , and the statistical performance of the regression model is shown in Table 2. We found the maximum negative and positive errors with 5'-HO-BDE-99 (experimental value 10.34 vs predicted value 9.59) and 6-HO-BDE-87 (8.44 vs 9.09), respectively. However, both of these values are smaller than the 2 -fold $\mathrm{rms}$
(0.427), indicating the consistency between experimental and predicted values.

Figure 1 shows that the predicted $-\log \mathrm{REC}_{20}$ is consistent with the experimental values, and Figure 2 indicates that with

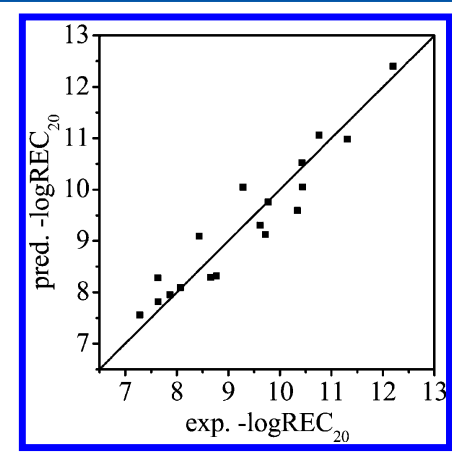

Figure 1. Predicted versus experimental $-\log \mathrm{REC}_{20}$ for $18 \mathrm{HO}$ PBDEs.

our developed model, there is no obvious dependence on experimental values for prediction errors and that no compound exceeds a 2 -fold rms.

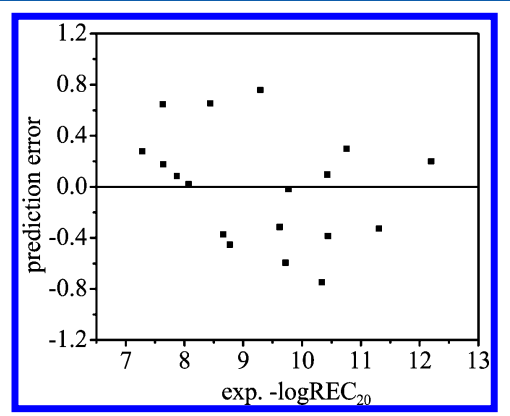

Figure 2. Prediction errors versus experimental $-\log \mathrm{REC}_{20}$ values for 18 HO-PBDEs.

To analyze the predictive performance for HO-PBDEs with a different number of bromine atoms, the rms values for subgroups with different numbers of bromine atoms are calculated: $r m s=0.180$ for dibrominated HO-PBDEs, and $0.269,0.445,0.549,0.282$ for tribrominated, tetrabrominated, pentabrominated, and hexabrominated HO-PBDEs, respectively. The class-specific rms values indicate that the prediction precision for tetra- and penta-substituted HO-PBDEs is significantly inferior to other HO-PBDE subsets. This observation indicates the need for further research when additional experimental data are available.

Model Interpretation. The regression model contains $Q E_{\text {occ }}$ and $Q E_{\text {vac }}$ evaluated at the hydroxyl oxygen atom and hydroxyl hydrogen atom, respectively, and $\log K_{\mathrm{ow}}$ that characterizes the partitioning behavior of the compounds between the biophase and water. The energy-limited donor charge $Q E_{\text {occ }}(-8.8 \mathrm{eV}, \mathrm{O})$ quantifies the amount of the donated electron charge associated with energy loss $(-8.8 \mathrm{eV})$ 
at the oxygen atom. An oxygen atom with a larger $Q E_{\text {occ }}$ value prefers to donate electron charge and, accordingly, can easily form a hydrogen bond with other hydrogen atoms as receptors. Because forming hydrogen bonds between HO-PBDEs and $\operatorname{TR} \beta$ is a key step for exerting thyroid hormone activity, ${ }^{20}$ it follows that increasing $Q E_{\text {occ }}$ correlates with increasing thyroid hormone activity, thus increasing - $\log \mathrm{REC}_{20}$. Therefore, $Q E_{\text {occ }}$ yields a positive regression coefficient in eq 6 (24.22).

The second descriptor is the energy-limited acceptor charge $Q E_{\text {vac }}(3.8 \mathrm{eV}, \mathrm{H})$. Converse to $Q E_{\text {occ }}$, which extracts electronic and energy information for occupied molecular orbitals, $Q E_{\mathrm{vac}}$ characterizes the ability of unoccupied orbitals to accept additional electron charges from specific sites, which can be interpreted as a localization of $E_{\mathrm{LUMO}}$. Thus, $Q E_{\mathrm{vac}}(3.8 \mathrm{eV}, \mathrm{H})$ quantifies the amount of accepted electron charge when the hydrogen atom gains $3.8 \mathrm{eV}$ energy. Given a constant $E_{\text {ref }}$ parameter (here, $3.8 \mathrm{eV}$ ), a larger $Q E_{\mathrm{vac}}$ at the hydrogen atom indicates that it could accept larger amounts of electron charge, thus tending to bind with atoms of high electronegativity. Thus, the HO-PBDE molecule with higher $Q E_{\mathrm{vac}}$ has a stronger binding ability with $\operatorname{TR} \beta$ and has a positive coefficient (2.61) in the regression model of $-\log \mathrm{REC}_{20}$.

$\log K_{\mathrm{ow}}$ is also selected in the regression model with a positive coefficient (0.79), indicating that the thyroid hormone activity of HO-PBDE molecules increases with $\log K_{\mathrm{ow}}$ values. Molecules with larger $\log K_{\text {ow }}$ prefer to partition into yeast cells; thus, they have more potency to disturb the activity of TR $\beta$ and have a larger $-\log \mathrm{REC}_{20}$ value.

Furthermore, the variable inflation factor (VIF) values are $1.222,1.727$, and 1.470 for $\log K_{\mathrm{ow}}, Q E_{\mathrm{occ}}(\mathrm{O})$, and $Q E_{\mathrm{vac}}(\mathrm{H})$, respectively, indicating that no multicollinearity exists, which is also reflected from the $t$-test result in which all parameters in the regression model are statistically significant at $\alpha=0.001$ level $(p<0.001)$.

Model Validation. As described above, two temporary subsets (70\% subset and 30\% subset) were randomly produced, both covering the $-\log \mathrm{REC}_{20}$ value range and chemical domain of the full dataset. Then, $70 \%$ of the compounds were recalibrated based on the descriptors in Table 1, yielding a new model to predict the $-\log \mathrm{REC}_{20}$ values of the remaining $30 \%$ of the compounds. The associated calibration and prediction statistics of this simulated external validation are listed in Table 2.

The $70 \%$ training subset yields a statistical result similar to that of the full data set, $r^{2}=0.888$, rms $=0.462$ vs $r^{2}=0.902$, and $\mathrm{rms}=0.427$. The new recalibration model also has regression coefficients similar to those of the original model, 25.85 for $Q E_{\text {occ }}(-8.8 \mathrm{eV}, \mathrm{O})$ on the $70 \%$ training subset and 24.22 on the full dataset, 2.82 vs 2.61 for $Q E_{\text {vac }}(3.8 \mathrm{eV}, \mathrm{H})$ and 0.70 vs 0.79 for $\log K_{\text {ow }}$. These results indicate that the developed model is statistically stable. The $30 \%$ testing subset has a performance comparable (and slightly superior) to that of the $70 \%$ subset and the full dataset, $q^{2}=0.921, \mathrm{rms}=0.362$. Again, the model appears to be statistically robust and has good predictive ability within the structural and $-\log \mathrm{REC}_{20}$ domain.

Then, the leave-10\%-out cross-validation was performed. Every time, $10 \%$ of the compounds remained to be predicted by the regression model based on $90 \%$ of the compounds, and this operation was repeated 10 times. The resulting $q^{2}$ equals 0.845 , and rms equals 0.536 , which remain similar to the statistical parameters of the calibration model, reflecting again that our model has a good degree of robustness and predictive power.
Influential Compounds and Outliers. Initially, the Kolmogorov-Smirnov test was applied to confirm that the prediction residuals of $-\log \mathrm{REC}_{20}$ followed normal distributions at the $95 \%$ confidence interval. Then, a Williams plot was drawn using leverage $h_{i}$ values and standardized residues (Figure 3). Clearly, all compounds were distributed at the left

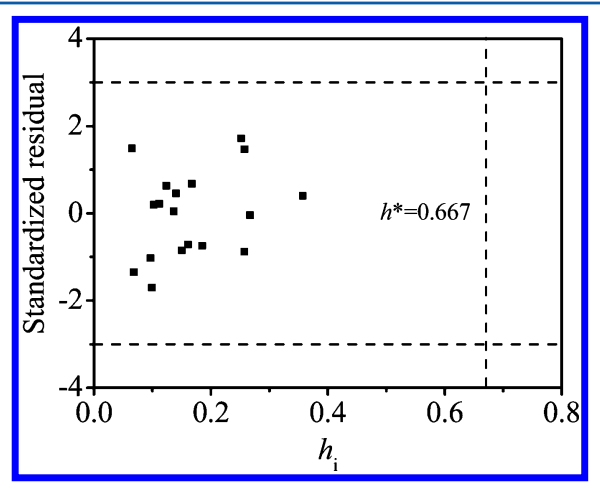

Figure 3. Williams plot with warning leverage $h^{*}=0.667$.

side of the Williams plot, i.e., all $h_{\mathrm{i}}$ values were smaller than the warning value $\left(h^{*}=0.667\right)$, indicating that the model has no influential compounds and that the dataset has great representativeness in descriptor space.

Furthermore, the standard residual is smaller than 3 (even smaller than 2) for every molecule, as shown in Figure 3. Therefore, there is no large gap between calibrated values and experimental values for the selected compounds; thus, there are no outliers. As shown in Scheme 1, our chemical domain consists of di-, tri-, tetra-, penta-, and hexabrominated monohydroxylated BDEs; therefore, the developed model could be applied to predict the thyroid hormone activity of these chemical congeners.

The regression model was developed based on experimental activity data, and the predictive results could also validate the experimental reliability to a certain extent. For example, the measured $-\log \mathrm{REC}_{20}$ for $4-\mathrm{HO}-\mathrm{BDE}-90$ is 7.63 , which is abnormally lower than the congeners with similar structures (9.29-10.44), and a very high positive predictive error $(0.65)$ is observed for this compound, implying that the experiment may underestimate its hormone activity (Table 1). Furthermore, the experimental data for two HO-BDE-28 and three HO-BDE-47 molecules show that the substitution of the hydroxyl group ortho to the $\mathrm{C}-\mathrm{O}-\mathrm{C}$ linkage could pose a larger thyroid disrupting effect than the meta substitution, with a difference of 0.79 and 0.65 , respectively (Table 1 ). However, the measurement value of $5^{\prime}$-HO-BDE-99 (meta-) is 10.34, which is markedly higher than 9.62 for 6'-HO-BDE-99 (ortho-), and 5'HO-BDE-99 shows the largest negative error in the regression model. Thus, its experimental hormone activity may also be somewhat doubtful. These indicate that in addition to clarifying the thyroid hormone activity mechanism of HO-PBDEs, an in silico study could also contribute to the quality diagnostics for the experimental data.

Comparison with Reference Results. The model performance was compared with reference results. For the same chemicals, there are two other studies that analyzed the interactions between HO-PBDE molecules and $\operatorname{TR} \beta$ and developed QSAR models. ${ }^{20,30}$ These research efforts reveal that the hydrogen bonding and electrostatic interactions are important molecular interactions, providing a theoretical basis 
Table 3. Statistical Performance of Different Methods for Predicting the $-\log \mathrm{REC}_{20}$ of HO-PBDEs

\begin{tabular}{|c|c|c|c|c|c|c|c|c|c|c|}
\hline \multirow[b]{2}{*}{ ref } & \multicolumn{4}{|c|}{ whole data set } & \multicolumn{3}{|c|}{ training set } & \multicolumn{3}{|c|}{ test set } \\
\hline & $n$ & $r^{2}$ & $q^{2}$ & $\mathrm{rms}$ & $n$ & $r^{2}$ & $\mathrm{rms}$ & $n$ & $q^{2}$ & $\mathrm{rms}$ \\
\hline this study & 18 & 0.902 & 0.902 & 0.427 & 13 & 0.888 & 0.462 & 5 & 0.845 & 0.427 \\
\hline 20 & 18 & 0.872 & 0.872 & 0.487 & 14 & 0.898 & 0.453 & 4 & 0.676 & 0.589 \\
\hline 30 & 18 & 0.936 & 0.934 & 0.349 & 14 & 0.951 & 0.099 & 4 & 0.813 & 0.448 \\
\hline
\end{tabular}

for the present investigation. The approach introduced in this study employs local molecular descriptors to extract electron and energy information for specific molecular sites relevant to the action center. As reflected by the regression parameters and mechanism analysis, the derived model could quantify the inherent electronic actions between HO-PBDEs and TR $\beta$, identify the significant structural features of HO-PBDEs for hormone activity, and explain their activity mechanism through the physicochemical meanings of the descriptors.

Because the statistical parameters in different studies are not uniform, we recalculated some parameters and summarized all of the statistics in Table 3. Compared with the model developed by Li et al., ${ }^{20}$ the present introduced model includes fewer descriptors ( 3 vs 6 ) and obtains better statistical results $\left(r^{2}=0.902, \mathrm{rms}=0.427\right.$ vs $\left.r^{2}=0.872, \mathrm{rms}=0.487\right)$ for the full dataset. The prediction performance for the test set also shows that our model has stronger robustness because $q^{2}$ is larger ( 0.845 vs 0.676$)$, and rms is smaller ( 0.427 vs 0.589 ).

On the basis of the comparison, the performance of our model appears inferior to 3D-QSAR ${ }^{30}$ (Table 3). However, although 3D-QSAR obtains excellent results for calibration, its prediction ability decreases significantly, reflected by $q^{2}=0.813$ and $\mathrm{rms}=0.448$ for the test set, which are inferior to those of our model. Furthermore, 3D-QSAR has no detailed equation expression but only shows the important molecular force fields for hormone reactivity, which is not beneficial for an in-depth explanation of the mechanism and for predictive applications.

The present developed model could capture intrinsic reactivity by employing local molecular descriptors and has superior robustness and predictive capability. This result will be helpful for in-depth investigations and accurate predictions of the thyroid hormone activities of other HO-PBDE compounds.

Ionization Effect on Hormone Activity. Because HOPBDEs contain ionizable functional groups, namely, $-\mathrm{OH}$, they could ionize depending on the $\mathrm{pH}$ of the environment and their $\mathrm{p} K_{\mathrm{a}}$ values. Yang et al. reported that the anionic phenolic compounds bind more strongly with human transthyretin (hTTR) than the corresponding neutral forms and found that the $\mathrm{p} K_{\mathrm{a}}$ is a very important parameter for quantifying the relative competing potency of the chemicals with $\mathrm{T} 4$ to hTTR $^{43}$ Therefore, we considered a similar question to determine whether the dissociation of HO-PBDEs affects their interaction with $\operatorname{TR} \beta$.

To determine the answer, we calculated $\mathrm{p} K_{\mathrm{a}}$ values using the commercial software ACD 12.0. Table S1 (Supporting Information) shows that $\mathrm{pK}$ a varies from 5.5 to 9.2. The $\mathrm{pH}$ value of the buffer solution in which experimental $-\log \mathrm{REC}_{20}$ data were obtained is 4.12 , and the endocellular $\mathrm{pH}$ of the yeast cell is approximately 5.5. Therefore, a large majority of the compounds maintain neutral forms at these $\mathrm{pH}$ values. Hence, dissociation has a slight effect on the hormone activity of the HO-PBDEs under these experimental conditions, which was also demonstrated by the poor negative correlation $\left(r^{2}=0.302\right)$ between $\mathrm{p} K_{\mathrm{a}}$ and $-\log \mathrm{REC}_{20}$. However, we should realize that the endocellular $\mathrm{pH}$ values of other cellular species or physiological $\mathrm{pH}$ values in organisms are higher than the $\mathrm{pH}$ in this experiment, normally $7.0-7.4$ (e.g., $\mathrm{pH} 7$ for HepG cells), which cause the dissociation for HO-PBDE compounds. However, such experimental data have been missing to date. The effect of molecular dissociation on thyroid hormone activity requires further experimental investigation to obtain more high-quality data for in silico studies.

Implications. In this study, the thyroid activity of $\mathrm{HO}$ PBDEs through the interaction with $\operatorname{TR} \beta$ was studied by an in silico approach. The molecular mechanism involved was analyzed in-depth via the electronic and energy properties of the chemicals. Because the interactions of xenobiotic compounds with biomacromolecules in the endocrine system are important molecular initiating events (MIEs) that incur endocrine disrupting effects, ${ }^{44}$ revealing the interactive mechanism of HO-PBDEs with $\mathrm{TR} \beta$ can help unveil the adverse outcome pathway (AOP). The predictive results could also verify the experimental reliability. Furthermore, the research approach is of great importance for investigating the interactions of HO-PBDEs with other biomacromolecules in the thyroid system, such as hTTR and the thyroid hormone sulfotransferase (SULT1A1), as well as with other endocrine systems.

Theoretically, there are 837 monohydroxylated BDEs and many chemicals with similar structures, e.g., HO-PCBs and HO-PCDDs. The predictive model that was developed will play an important role in virtual screening to identify compounds that potentially interact with $\operatorname{TR} \beta$ and disrupt thyroid hormone activity. The predictive model could also assist with "green chemistry" in designing products with low toxicity.

\section{ASSOCIATED CONTENT}

\section{S Supporting Information}

Calculated $\mathrm{pK}$ a values for HO-PBDEs. The Supporting Information is available free of charge on the ACS Publications website at DOI: 10.1021/acs.chemrestox.5b00127.

\section{AUTHOR INFORMATION}

\section{Corresponding Authors}

*(H.Y.) Tel: +86-579-82282273. E-mail: yhy@zjnu.cn.

*(L.J.) Tel: +86-571-88982467. E-mail: jilienv@zju.edu.cn.

\section{Funding}

This research was financially supported by National Natural Science Foundation of China (21207119) and Foundation of Zhejiang Educational Committee (Y201226156), which are gratefully acknowledged.

\section{Notes}

The authors declare no competing financial interest.

\section{ABBREVIATIONS}

AOP, adverse outcome pathway; AOs, atomic orbitals; bias, systematic error; $E E_{\text {occ }}\left(E_{\text {ref }} r\right)$, energy-weighted donor energy; $E E_{\mathrm{vac}}\left(E_{\mathrm{ref}}, r\right)$, energy-weighted acceptor energy; $E Q_{\mathrm{occ}}(q, r)$, charge-limited donor energy; $E Q_{\mathrm{vac}}(q, r)$, charge-limited 
acceptor energy; $h_{\mathrm{j}}$ leverage value; $h^{*}$, warning leverage value; HOMO, the highest occupied molecular orbital; HO-PBDEs, hydroxylated polybrominated diphenyl ethers; hTTR, human transthyretin; LCAO-MO, linear combination of atomic orbitals to molecular orbitals; $\log K_{\text {ow }}$ n-octanol/water partition coefficient; log $\mathrm{REC}_{20}$, minus logarithm of the chemical concentration inducing $20 \%$ of the maximum effect; me, mean error; MIEs, molecular initiating events; MLR, multilinear regression; mne, maximum negative error; MOs, molecular orbitals; mpe, maximum positive error; PBDEs, polybrominated diphenyl ethers; $\mathrm{p} K_{\mathrm{a}}$ dissociation constant; $q^{2}$, predictive squared correlation coefficient; $Q E_{\text {occ }}(\varepsilon, r)$, energylimited donor charge; $Q E_{\mathrm{vac}}(\varepsilon, r)$, energy-limited acceptor charge; QSAR, quantitative structure-activity relationship; $r^{2}$, squared correlation coefficient; rms, root-mean-square error; SULT1A1, sulfotransferase; $\mathrm{T}_{4}$, 3,3',5,5' -tetraiodo-L-thyronine; THs, thyroid hormones; TR $\alpha$, thyroid hormone receptor alpha; $\operatorname{TR} \beta$, thyroid hormone receptor beta; TRs, thyroid hormone receptors; TTR, transthyretin; VIF, variable inflation factor; $y_{i}^{\text {fit }}$, regression-fitted value; $y^{\text {mean }}$, observed mean value; $y_{i}^{\text {obs }}$, observed value; $y_{i}^{\text {pred }}$, predicted value

\section{REFERENCES}

(1) Birnbaum, L. S., and Staskal, D. F. (2004) Brominated flame retardants: cause for concern? Environ. Health Perspect. 112, 9-17.

(2) Boon, J. P., Lewis, W. E., Tjoen, A. C. M. R, Allchin, C. R., Law, R. J., De Boer, J., Ten Hallers-Tjabbes, C. C., and Zegers, B. N. (2002) Levels of polybrominated diphenyl ether (PBDE) flame retardants in animals representing different trophic levels of the North Sea food Web. Environ. Sci. Technol. 36, 4025-4032.

(3) Dodder, N. G., Strandberg, B., and Hites, R. A. (2002) Concentrations and spatial variations of polybrominated diphenyl ethers and several organochlorine compounds in fishes from the northeastern United States. Environ. Sci. Technol. 36, 146-151.

(4) Mazdai, A., Dodder, N. G., Abernathy, M. P., Hites, R. A., and Bigsby, R. M. (2003) Polybrominated diphenyl ethers in maternal and fetal blood samples. Environ. Health Perspect. 111, 1249-1252.

(5) Stapleton, H. M., Eagle, S., Anthopolos, R., Wolkin, A., and Miranda, M. L. (2011) Associations between polybrominated diphenyl ether (PBDE) flame retardants, phenolic metabolites, and thyroid hormones during pregnancy. Environ. Health Perspect. 119, 14541459.

(6) Harley, K. G., Marks, A. R., Chevrier, J., Bradman, A., Sjodin, A., and Eskenazi, B. (2010) PBDE concentrations in women's serum and fecundability. Environ. Health Perspect. 118, 699-704.

(7) Stapleton, H. M., Kelly, S. M., Pei, R., Letcher, R. J., and Gunsch, C. (2009) Metabolism of polybrominated diphenyl ethers (PBDEs) by human hepatocytes in vitro. Environ. Health Perspect. 117, 197-202.

(8) Kojima, H., Takeuchi, S., Uramaru, N., Sugihara, K., Yoshida, T., and Kitamura, S. (2009) Nuclear hormone receptor activity of polybrominated diphenyl ethers and their hydroxylated and methoxylated metabolites in transactivation assays using Chinese hamster ovary cells. Environ. Health Perspect. 117, 1210-1218.

(9) Raff, J. D., and Hites, R. A. (2006) Gas-phase reactions of brominated diphenyl ethers with $\mathrm{OH}$ radicals. J. Phys. Chem. A 110, 10783-10792.

(10) Ueno, D., Darling, C., Alaee, M., Pacepavicius, G., Teixeira, C., Campbell, L., Letcher, R. J., Bergman, A., Marsh, G., and Muir, D. (2008) Hydroxylated polybrominated diphenyl ethers (OH-PBDEs) in the abiotic environment: surface water and precipitation from Ontario, Canada. Environ. Sci. Technol. 42, 1657-1664.

(11) Malmvarn, A., Marsh, G., Kautsky, L., Athanasiadou, M., Bergman, A., and Asplund, L. (2005) Hydroxylated and methoxylated brominated diphenyl ethers in the red algae Ceramium tenuicorne and blue mussels from the Baltic Sea. Environ. Sci. Technol. 39, 2990-2997.

(12) Marsh, G., Athanasiadou, M., Bergman, A., and Asplund, L. (2004) Identification of hydroxylated and methoxylated polybromi- nated diphenyl ethers in Baltic Sea salmon (Salmo salar) blood. Environ. Sci. Technol. 38, 10-18.

(13) Valters, K., Li, H., Alaee, M., D’Sa, I., Marsh, G., Bergman, A., and Letcher, R. J. (2005) Polybrominated diphenyl ethers and hydroxylated and methoxylated brominated and chlorinated analogues in the plasma of fish from the Detroit River. Environ. Sci. Technol. 39, $5612-5619$.

(14) Verreault, J., Gabrielsen, G. W., Chu, S., Muir, D. C., Andersen, M., Hamaed, A., and Letcher, R. J. (2005) Flame retardants and methoxylated and hydroxylated polybrominated diphenyl ethers in two Norwegian Arctic top predators: glaucous gulls and polar bears. Environ. Sci. Technol. 39, 6021-6028.

(15) Mizukawa, H., Nomiyama, K., Nakatsu, S., Yachimori, S., Hayashi, T., Tashiro, Y., Nagano, Y., and Tanabe, S. (2013) Speciesspecific differences in the accumulation features of organohalogen contaminants and their metabolites in the blood of Japanese terrestrial mammals. Environ. Pollut. 174, 28-37.

(16) Qiu, X., Bigsby, R. M., and Hites, R. A. (2009) Hydroxylated metabolites of polybrominated diphenyl ethers in human blood samples from the United States. Environ. Health Perspect. 117, 93-98.

(17) Lacorte, S., and Ikonomou, M. G. (2009) Occurrence and congener specific profiles of polybrominated diphenyl ethers and their hydroxylated and methoxylated derivatives in breast milk from Catalonia. Chemosphere 74, 412-420.

(18) Zhou, J., Chen, J., Liang, C.-H., Xie, Q., Wang, Y., and Zhang, S. (2011) Quantum chemical investigation on the mechanism and kinetics of PBDE photooxidation by $\cdot \mathrm{OH}$ : A case study for BDE-15. Environ. Sci. Technol. 45, 4839-4845.

(19) Wang, X., Wang, Y., Chen, J., Ma, Y., Zhou, J., and Fu, Z. (2012) Computational toxicological investigation on the mechanism and pathways of xenobiotics metabolized by cytochrome P450: A case of BDE-47. Environ. Sci. Technol. 46, 5126-5133.

(20) Li, F., Xie, Q., Li, X., Li, N., Chi, P., Chen, J., Wang, Z., and Hao, C. (2010) Hormone activity of hydroxylated polybrominated diphenyl ethers on human thyroid receptor-beta: in vitro and in silico investigations. Environ. Health Perspect. 118, 602-606.

(21) Zhou, T., Taylor, M. M., DeVito, M. J., and Crofton, K. M. (2002) Developmental exposure to brominated diphenyl ethers results in thyroid hormone disruption. Toxicol. Sci. 66, 105-116.

(22) Qiu, X., Mercado-Feliciano, M., Bigsby, R. M., and Hites, R. A. (2007) Measurement of polybrominated diphenyl ethers and metabolites in mouse plasma after exposure to a commercial pentabromodiphenyl ether mixture. Environ. Health Perspect. 115, $1052-1058$

(23) Darnerud, P. O. (2003) Toxic effects of brominated flame retardants in man and in wildlife. Environ. Int. 29, 841-853.

(24) Butt, C. M., and Stapleton, H. M. (2013) Inhibition of thyroid hormone sulfotransferase activity by brominated flame retardants and halogenated phenolics. Chem. Res. Toxicol. 26, 1692-1702.

(25) Cao, J., Lin, Y., Guo, L., Zhang, A., Wei, Y., and Yang, Y. (2010) Structure-based investigation on the binding interaction of hydroxylated polybrominated diphenyl ethers with thyroxine transport proteins. Toxicology 277, 20-28.

(26) Kitamura, S., Shinohara, S., Iwase, E., Sugihara, K., Uramaru, N., Shigematsu, H., Fujimoto, N., and Ohta, S. (2008) Affinity for thyroid hormone and estrogen receptors of hydroxylated polybrominated diphenyl ethers. J. Health Sci. 54, 607-614.

(27) Wan, Y., Choi, K., Kim, S., Ji, K., Chang, H., Wiseman, S., Jones, P. D., Khim, J. S., Park, S., Park, J., Lam, M. H., and Giesy, J. P. (2010) Hydroxylated polybrominated diphenyl ethers and bisphenol $\mathrm{A}$ in pregnant women and their matching fetuses: placental transfer and potential risks. Environ. Sci. Technol. 44, 5233-5239.

(28) Harvey, C. B., and Williams, G. R. (2002) Mechanism of thyroid hormone action. Thyroid 12, 441-446.

(29) Forrest, D., and Vennstrom, B. (2000) Functions of thyroid hormone receptors in mice. Thyroid 10, 41-52.

(30) Li, X., Ye, L., Wang, X., Wang, X., Liu, H., Zhu, Y., and Yu, H. (2012) Combined 3D-QSAR, molecular docking and molecular dynamics study on thyroid hormone activity of hydroxylated 
polybrominated diphenyl ethers to thyroid receptors beta. Toxicol. Appl. Pharmacol. 265, 300-307.

(31) Karelson, M., Lobanov, V. S., and Katritzky, A. R. (1996) Quantum-chemical descriptors in QSAR/QSPR studies. Chem. Rev. 96, 1027-1044.

(32) Klamt, A. (1993) Estimation of gas-phase hydroxyl radical rate constants of organic-compounds from molecular-orbital calculations. Chemosphere 26, 1273-1289.

(33) Klamt, A. (1996) Estimation of gas-phase hydroxyl radical rate constants of oxygenated compounds based on molecular orbital calculations. Chemosphere 32, 717-726.

(34) Schwöbel, J., Ebert, R.-U., Kühne, R., and Schüürmann, G. (2009) Prediction of the intrinsic hydrogen bond acceptor strength of organic compounds by local molecular parameters. J. Chem. Inf. Model. 49, 956-962.

(35) Schwöbel, J., Ebert, R.-U., Kühne, R., and Schüürmann, G. (2009) Modeling the $\mathrm{H}$ bond donor strength of $-\mathrm{OH},-\mathrm{NH}$, and $-\mathrm{CH}$ sites by local molecular parameters. J. Comput. Chem. 30, 1454-1464. (36) Böhnhardt, A., Kühne, R., Ebert, R.-U., and Schüürmann, G. (2010) Predicting rate constants of $\mathrm{OH}$ radical reactions with organic substances - advances for oxygenated organics through a molecular orbital HF/6-31G** Approach. Theor. Chem. Acc. 127, 355-367.

(37) Yu, H., Kühne, R., Ebert, R.-U., and Schüürmann, G. (2010) Comparative analysis of QSAR models for predicting $\mathrm{pK} K_{\mathrm{a}}$ of organic oxygen acids and nitrogen bases from molecular structure. J. Chem. Inf. Model. 50, 1949-1960.

(38) Wondrousch, D., Böhme, A., Thaens, D., Ost, N., and Schuiürmann, G. (2010) Local electrophilicity predicts the toxicityrelevant reactivity of michael acceptors. J. Phys. Chem. Lett. 1, 16051610.

(39) Frisch, M. J. T., Trucks, G. W., Schlegel, H. B., Scuseria, G. E., Robb, M. A., Cheeseman, J. R., Montgomery, J. A., Vreven, T., Kudin, K. N., Burant, J. C., Millam, J. M., Iyengar, S. S., Tomasi, J., Barone, V., Mennucci, B., Cossi, M., Scalmani, G., Rega, N., Petersson, G. A., Nakatsuji, H., Hada, M., Ehara, M., Toyota, K., Fukuda, R., Hasegawa, J., Ishida, M., Nakajima, T., Honda, Y., Kitao, O., Nakai, H., Klene, M., Li, X., Knox, J. E., Hratchian, H. P., Cross, J. B., Adamo, C., Jaramillo, J., Gomperts, R., Stratmann, R. E., Yazyev, O., Austin, A. J., Cammi, R., Pompelli, C., Ochterski, J. W., Ayala, P. Y., Morokuma, K., Voth, G. A., Salvador, P., Dannenberg, J. J., Zakrzewski, V. G., Dapprich, S., Daniel, A. D., Strain, M. C., Farkas, O., Malick, D. K., Rabuck, A. D., Raghavavachari, K., Foresman, J. B., Ortiz, J. V., Cui, Q., Baboul, A. G., Clifford, S., Cioslowski, J., Stefanov, B. B., Liu, G., Liashenko, A., Piskorz, P., Komaromi, I., Martin, R. L., Fox, D. J., Keith, T., Al-Laham, M. A., Peng, C., Nanayakkara, A., Challacombe, M., Gill, P. M. W., Johnson, B., Chen, W., Wong, M., Gonzalez, C., and Pople, J. A. (2003) Gaussian 03, revision C.02, Gaussian Inc., Pittsburgh, PA.

(40) (2012) Estimation Programs Interface Suite for Microsoft ${ }^{\circledR}$ Windows, v 4.10, United States Environmental Protection Agency, Washington, DC.

(41) Schüürmann, G., Ebert, R.-U., Chen, J., Wang, B., and Kühne, R. (2008) External validation and prediction employing the predictive squared correlation coefficient - Test set activity mean vs training set activity mean. J. Chem. Inf. Model. 48, 2140-2145.

(42) Chatterjee, S., and Hadi, A. S. (1986) Influential observations, high leverage points, and outliers in linear regression. Stat. Sci. 1, 379416.

(43) Yang, X., Xie, H., Chen, J., and Li, X. (2013) Anionic phenolic compounds bind stronger with transthyretin than their neutral forms: Nonnegligible mechanisms in virtual screening of endocrine disrupting chemicals. Chem. Res. Toxicol. 26, 1340-1347.

(44) Vinken, M. (2013) The adverse outcome pathway concept: A pragmatic tool in toxicology. Toxicology 312, 158-165. 